\title{
Risk of atrial fibrillation in athletes: a systematic review and meta-analysis
}

\author{
William Newman, ${ }^{1}$ Gemma Parry-Williams, ${ }^{2}$ Jonathan Wiles, ${ }^{1}$ Jamie Edwards, ${ }^{1}$ \\ Sabina Hulbert, ${ }^{3}$ Konstantina Kipourou, ${ }^{4}$ Michael Papadakis, ${ }^{2}$ Rajan Sharma, ${ }^{4}$ \\ Jamie O'Driscoll (1) 1,4
}

\begin{abstract}
- Additional supplemental material is published online only. To view, please visit the journal online (http://dx.doi. org/10.1136/bjsports-2021103994).
\end{abstract}

${ }^{1}$ School of Psychology and Life Sciences, Canterbury Christ Church University, Canterbury, Kent, UK

${ }^{2}$ Cardiology Clinical Academic Group, St George's University of London, London, UK ${ }^{3}$ School of Psychology, Politics and Sociology, Canterbury Christ Church University, Canterbury, Kent, UK ${ }^{4}$ Depertment of Cardiology, St George's University Hospitals NHS Foundation Trust, London, UK

\section{Correspondence to} Dr Jamie O'Driscoll, School of Human and Life Sciences, Canterbury Christ Church University, Canterbury CT1 1QU, UK;

jamie.odriscoll@canterbury. ac.uk

Accepted 11 June 2021 Published Online First 12 July 2021

\section{ABSTRACT}

Objective A systematic review, meta-analysis and meta-regression were performed on selected studies to investigate the incidence of atrial fibrillation (AF) among athletes compared with non-athlete controls.

Design Meta-analysis with heterogeneity analysis and subsequent meta-regression to model covariates were performed. The mode of exercise (endurance and mixed sports) and age were the a priori determined covariates. Data sources PubMed, MEDLINE, Science Direct, SPORTDiscus and the Cochrane library were searched. Eligibility criteria Research articles published after 1990 and before 2 December 2020 were included if they reported the number of AF cases in athletes with non-athlete (physically active or inactive) control groups, were case-control or cohort studies and if data allowed calculation of OR.

Results The risk of developing AF was significantly higher in athletes than in non-athlete controls (OR: 2.46; $95 \% \mathrm{Cl} 1.73$ to $3.51 ; p<0.001, Z=4.97)$. Mode of exercise and risk of AF were moderately correlated ( $B=0.1259, p=0.0193)$, with mixed sport conferring a greater risk of $A F$ than endurance sport $(B=-0.5476$, $p=0.0204)$. Younger ( $<55$ years) athletes were significantly more likely to develop AF compared with older ( $\geq 55$ years) athletes $(B=-0.02293, p<0.001)$. Conclusion Athletes have a significantly greater likelihood of developing AF compared with non-athlete controls, with those participating in mixed sport and younger athletes at the greatest risk. Future studies of AF prevalence in athletes according to specific exercise dose parameters, including training and competition history, may aid further in delineating those at risk.

\section{INTRODUCTION}

Physical activity and exercise training reduce metabolic disease risk, ${ }^{1}$ improve cardiovascular health ${ }^{2}$ and mental well-being ${ }^{3}$ and are associated with healthy ageing. ${ }^{4}$ Studies also suggest that exercise can reduce the burden of arrhythmias, such as atrial fibrillation (AF), ${ }^{5}$ which is the most common cardiac arrhythmia worldwide. Indeed, the prevalence of AF in the general population is as high as $3.3 \%{ }^{7}$ and is associated with a 1.5 -fold increased mortality risk, most commonly secondary to cerebrovascular events. ${ }^{8}$

AF is also the most prevalent arrhythmia in athletic populations. It has been reported that male endurance athletes performing high-intensity and high-volume exercise training are at an increased risk, ${ }^{9}$ with studies suggesting a U-shaped curve, with lack of physical activity and long-term vigourous exercise both increasing the risk of developing AF. ${ }^{10} 11$ The mechanisms by which exercise training increases the risk of AF are complex and speculative, ${ }^{12}$ but may include atrial dilation, adrenergic activation, vagal tone, chronic inflammation, pulmonary foci and interstitial fibrosis, occuring as a result of excessive strain through augmented cardiac output and atrial stretch. ${ }^{13}$

Two prior meta-analyses investigating the possible increased risk of AF in athletes have demonstrated equivocal findings, with ORs of $1.64^{14}$ and 5.29. ${ }^{15}$ Interestingly, Ayinde $e t a l^{15}$ identified age as a significant determinant of AF risk, with younger athletes (below 40 years old) at greater relative risk than athletes $>54$ years of age. However, these metaanalyses excluded studies of athletes with hypertension or diabetes, which potentially restricts the availability of larger pooled study data for analysis. As such, this meta-analysis aims to establish the risk of AF in athletes and further elucidate the influence of sport type and cardiovascular disease (CVD) risk factors on the risk of developing AF. As the prevalence is relatively low, this paper aimed to perform a meta-analysis and pool multiple study data to draw a more conclusive overall prevalence among athletes.

\section{METHODS}

\section{Search strategy}

This systematic review and meta-analysis was fully compliant with and performed according to the Preferred Reporting Items for Systematic Reviews and Meta-Analyses (PRISMA) guidelines, ${ }^{16}$ including use of the described PICOS method for the systematic review process. Figure 1 details the PRISMA systematic review flowchart.

PubMed, MEDLINE, Science Direct, the Cochrane library and SPORTDiscus online databases were searched for studies reporting AF or atrial flutter in athletes in journal articles published before 2 December 2020. Studies written in English and published from 1990 onwards were accepted. Key Boolean search terms were utilised to identify relevant studies and included; 'atrial fibrillation' OR 'atrial flutter' AND 'athlete', 'sport', 'endurance' AND 'exercise'. Where possible, corresponding study authors were contacted to ascertain whether non-published data was available or in the pre-print stage, and studies found through the systematic protocol were screened for exterior citations and their respective reference lists searched for eligible studies. 


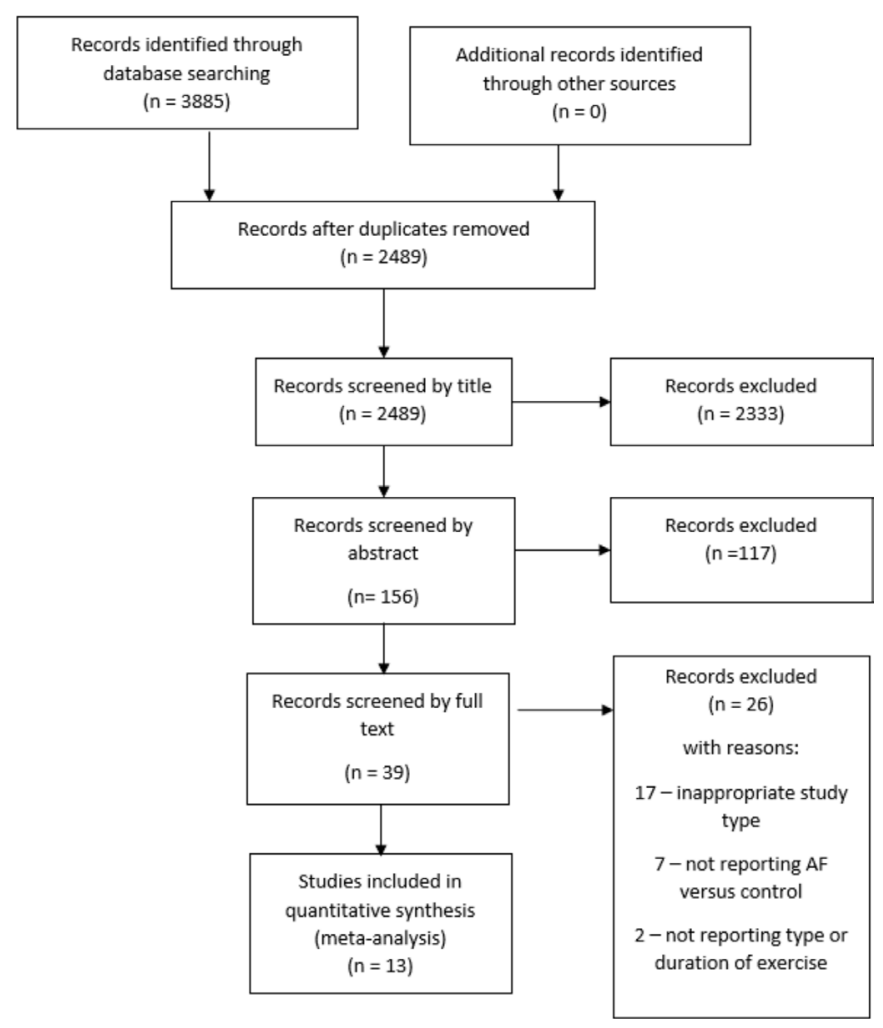

Figure 1 Preferred Reporting Items for Systematic Reviews and MetaAnalyses (PRISMA) systematic review and meta-analysis flowchart/ decision tree. $A F$, atrial fibrillation.

\section{Study eligibility}

Screening was carried out following the exclusion of duplicate articles. Studies were initially excluded by title and then by abstract. Studies retained for the next step of evaluation were screened and included if: they reported the number of AF cases in athletes with non-athlete control groups, were case-control or cohort study design and the presentation of data was in the form of ORs, or at least data whereby OR and CIs could be precisely calculated.

Studies were excluded in the next evaluation step if they did not include a control group and participants older than 18 years who had performed regular exercise training for at least a 2-year period prior to screening. For the purpose of this meta-analysis, 'athletes' were defined according to the European Society of Cardiology classification. ${ }^{17}$ Studies of athlete's participating in sports other than the following were excluded; cycling, running, swimming, Nordic skiing, orienteering, rowing (endurance sports) and mixed sports (football, netball, rugby, etc) as defined by Pelliccia et al. ${ }^{17}$ Studies where participants had concurrent diseases were excluded except for hypertension and diabetes, to exclude other confounding causes of AF but to include conditions common among the general population. ${ }^{18}$ Studies with these two concurrent diseases were classified and grouped for further subgroup analysis to determine if these diseases moderate the degree of AF prevalence and effect. Furthermore, studies reporting atrial flutter were included due to it being a precursor to AF.

For this meta-analysis, we included studies with non-exercising or recreationally exercising controls versus athlete populations to ascertain whether an optimal exercise duration, intensity or mode exists before AF manifests versus the general population. Non-athletic leisurely activity and physical activity participants were defined as physically active individuals without training regimes, athletic ambitions or participation in regular competition. In the literature, this has been regarded as 'leisure time physical activity'. This type of physical activity is generally at or below national physical activity guidelines, but not near the volumes or intensities seen through purposed exercise training undertaken by athletes.

\section{Data extraction, coding and study quality}

Our initial search identified 3885 citations. After all exclusions were applied, 13 studies were included and proceeded through to the data extraction and coding step (figure 1). The 13 studies included in the meta-analysis (table 1) were coded and

\begin{tabular}{|c|c|c|c|c|c|c|c|}
\hline Author & Year & Country & Sample size & Average age (years) & Sport type & Study design & Risk of bias* \\
\hline Aizer et al & 2009 & America & 8448 & 52 & Mixed and Endurancet & Cohort & 2 \\
\hline Andersen et al & 2013 & Sweden & 52755 & 57 & Nordic Skiing & Cohort & 2 \\
\hline Baldesberger et al & 2008 & Switzerland & 124 & 67 & Cycling & Cohort & 2 \\
\hline Calvo et al & 2016 & Spain & 172 & 46 & Mixed and Enduranceł & Case-control & 1 \\
\hline Claessen et al & 2011 & Belgium & 156 & 52 & Mixed and Endurance§ & Case-control & 2 \\
\hline Elosua et al & 2006 & Spain & 160 & 43 & Mixed and Endurance & Case-control & 1 \\
\hline Karjalainen et al & 1998 & Finland & 440 & 46 & Orienteering & Cohort & 1 \\
\hline Molina et al & 2008 & Spain & 473 & 39 & Running & Cohort & 1 \\
\hline Mont et al & 2002 & Spain & 216 & 44 & Mixed and Endurance ${ }^{* *}$ & Case-control & 2 \\
\hline Myrstad et al & 2014 & Norway & 2376 & 69 & Nordic Skiing & Cohort & 2 \\
\hline Myrstad et al & 2016 & Norway & 4952 & 69 & Nordic Skiing & Cohort & 2 \\
\hline Schreiner et al & 2016 & America & 149 & 72 & Swimming & Case-control & 3 \\
\hline Van Buuren et al & 2012 & Germany & 57 & 57 & Handball & Case-control & 2 \\
\hline
\end{tabular}

References are available in the supplemental file.

*Risk of bias: $1=$ low, $2=$ moderately low, $3=$ moderately high, $4=$ high. See online supplemental file 1 for full references.

tCycling, racquet sports and swimming.

‡Cycling, gym, marathon running, swimming, team sports, trekking and other.

§Basketball, cycling, diving, handball, horse riding, running, soccer, tennis, triathlon.

ףUnknown sports.

${ }^{* *}$ Athletics, cycling, running, soccer, swimming, weight lifting. 
respective data was extracted including systematic processing of study details, country of registration, year of publication, journal impact statistics and full patient anthropometric and clinical variables, where available. Study data was extracted and a risk of bias analysis performed, which were systematically screened by a second reviewer. When disputes were detected in coding or quality analyses, the reviewers met to discuss any conflicts, which were then resolved and evaluated further.

AF OR, relative risks and raw data were extracted, and OR was calculated according to the presence of sample size, control and athlete AF diagnosis data. The adjusted status and variables adjusted for in each study are provided in the (online supplemental table S1). When insufficient data was detected, best efforts were made to contact the relevant authors of the study or to seek online supplemental data. If this data was not available, these studies were then excluded from the rest of the analyses.

Qualitative risk of bias assessment was carried out utilising the tool from CLARITY at McMaster University (Evidence Partners, 2019) to ascertain the risk of bias in the cohort and case-control studies independently. For each of the outcomes, a number was assigned to the qualitative descriptor outcomes. The questions included queries surrounding the sampling of the population, composition and extraction of clinical data, and other factors surrounding blinding of participants and researchers. For case-control studies, there were five questions, whereas eight questions were asked for cohort studies. The response to the questions was completed using Likert integers; for example, in the question 'can we be confident in the assessment of the exposure', $1=$ definitely yes (low risk of bias) and $5=$ definitely no (high risk of bias). The overall results of the study quality assessment composed of a single numerical value resulting from an average of the individual question outcomes. Further quantitative study quality metrics not addressed by qualitative means were computed alongside the meta-analysis utilising funnel plot analyses and asymmetry testing with tests of heterogeneity.

\section{Data analysis and research synthesis}

As aforementioned, raw data was extracted from studies and transformed into OR with 95\% CIs. Comprehensive MetaAnalysis (Comprehensive Meta-Analysis V.3, Biostat, Englewood, New Jersey, USA) was used to assess the reported cases of AF in athletes versus non-athletes. A random effects pooled analysis was performed on the 13 final studies due to the interstudy variability with case-control and cohort studies, and the assessment of $\mathrm{AF}$ risk in all athletes from a population, allowing conclusions of a more general level of inference. ${ }^{19}$ Furthermore, random effects models are typically selected when interstudy variability is confirmed through significant heterogeneity. ${ }^{20}$ The results of the pooled analysis were considered significant when a $p$ value of $<0.05$ was present and the test $Z$ statistic was $>2$. Meta-analyses were also performed independently on the following conditions; age, comorbid CVD risk factor inclusion, study quality and study design, to investigate pooled effects.

Studies were inputted systematically, allowing the individual weightings of each study to be visible and the weighted effect on the overall effect size of the pooled analysis discerned for each study (online supplementary table S2). Heterogeneity was tested alongside the pooled analysis to quantitatively assess the study quality and risk of bias using the $\chi^{2}$ technique and reported as the $\mathrm{I}^{2}$ statistic. If the $\mathrm{I}^{2}$ statistic was $>40 \%$, it was considered significant. ${ }^{20}$ Once past this threshold, post hoc tests such as Egger's test (1997) were systematically planned to qualitatively assess the presence of funnel plot asymmetry, suggesting publication bias.

A meta regression was run to ascertain if any effect moderator variables influenced the risk of developing $\mathrm{AF}$ in athletes versus non-athletes. The planned moderators to be assessed independently were presence of diabetes, presence of hypertension, number of cases of high cholesterol, cases of cigarette smoking, body mass index, mode of exercise, age, sport type and study design.

\section{RESULTS}

\section{Study and participant characteristics}

The meta-analysis included 13 studies (7 cohort and 6 case-control) with a pooled sample size of 70478 participants, which included 63662 controls and 6816 athletes (online supplemental file). Five studies included athletes with the CVD risk factors diabetes and/or hypertension. Risk of bias scores and sport types are presented in table 1 . Only two studies provided data on volume or average duration of exercise and both defined this by lifetime exercise hours and could therefore not be used to quantify weekly exposure.

\section{Primary analysis}

The initial random effects pooled analysis demonstrated that the risk of AF was significantly higher in athletes than nonathletes (OR: 2.46; 95\% CI 1.73 to 3.51, $\mathrm{p}<0.001, \mathrm{Z}=4.97$ ) (figure 2). There was significant heterogeneity among the studies $\left(\mathrm{p}<0.001, \mathrm{I}^{2}=84 \%\right)$. The post hoc Egger's test (1997) was statistically significant $(\mathrm{p}<0.001)$, suggesting publication bias.

\section{Subgroup analysis}

When dichotomising studies into those with and without CVD risk factors (type 2 diabetes mellitus and hypertension), there was no significant difference in the relative risk of $\mathrm{AF}$ in athletes with CVD risk factors versus non-athletes with CVD risk factors (OR: $1.51 ; 95 \%$ CI 0.90 to $2.54, \mathrm{p}=0.116, \mathrm{Z}=1.572$ ). However, in athletes and non-athletes without CVD risk factors, athletes had a significantly greater relative risk of $\mathrm{AF}(\mathrm{OR}: 3.66 ; 95 \% \mathrm{CI}$ 2.28 to $5.88, \mathrm{p}<0.001, \mathrm{Z}=5.355$ ). Younger athletes (OR: 3.60; $95 \%$ CI 2.09 to $6.29, \mathrm{p}<0.001, \mathrm{Z}=4.603$ ) had a significantly higher relative risk of $\mathrm{AF}$ than older athletes (OR: $1.76 ; 95 \% \mathrm{CI}$ 0.97 to $3.21, \mathrm{p}=0.065, \mathrm{Z}=1.845$ ) (figure 3 ) and good quality studies (OR: 2.32; 95\% CI 1.62 to $3.32, \mathrm{p}<0.001, \mathrm{Z}=4.610$ ) reported lower overall $\mathrm{OR}$ for $\mathrm{AF}$ in athletes than poor quality studies (OR: 4.80; 95\% CI 1.22 to $18.88, \mathrm{p}=0.025, \mathrm{Z}=2.244$ ) (online supplemental figure S2). Case-control studies (OR: 4.91; $95 \%$ CI 3.48 to $6.93, \mathrm{p}<0.001, \mathrm{Z}=9.03$ ) produced a greater relative risk of $\mathrm{AF}$ in athletes than cohort-design studies (OR: $1.26 ; 95 \%$ CI 1.02 to $1.56, p=0.035, Z=2.11$ ). However, it was not possible to appropriately analyse the association of exercise intensity and AF risk due to insufficient data.

\section{Moderator analysis}

There was a significant relationship between the mode of exercise and risk of $\mathrm{AF}(\mathrm{B}=0.1259, \mathrm{p}=0.0193)$, with mixed sport conferring a greater risk of AF compared with endurance sport $(B=-0.5476, p=0.0204)$. Of the endurance sports, cycling conferred the highest risk of $\mathrm{AF}$ and Nordic skiing conferred the lowest risk (see online supplemental figure S3 for the lowest to highest risk of AF in endurance sports). Furthermore, younger ( $<55$ years) athletes were more likely to develop AF $(B=-0.02293, p<0.001)$. There was a significant relationship 


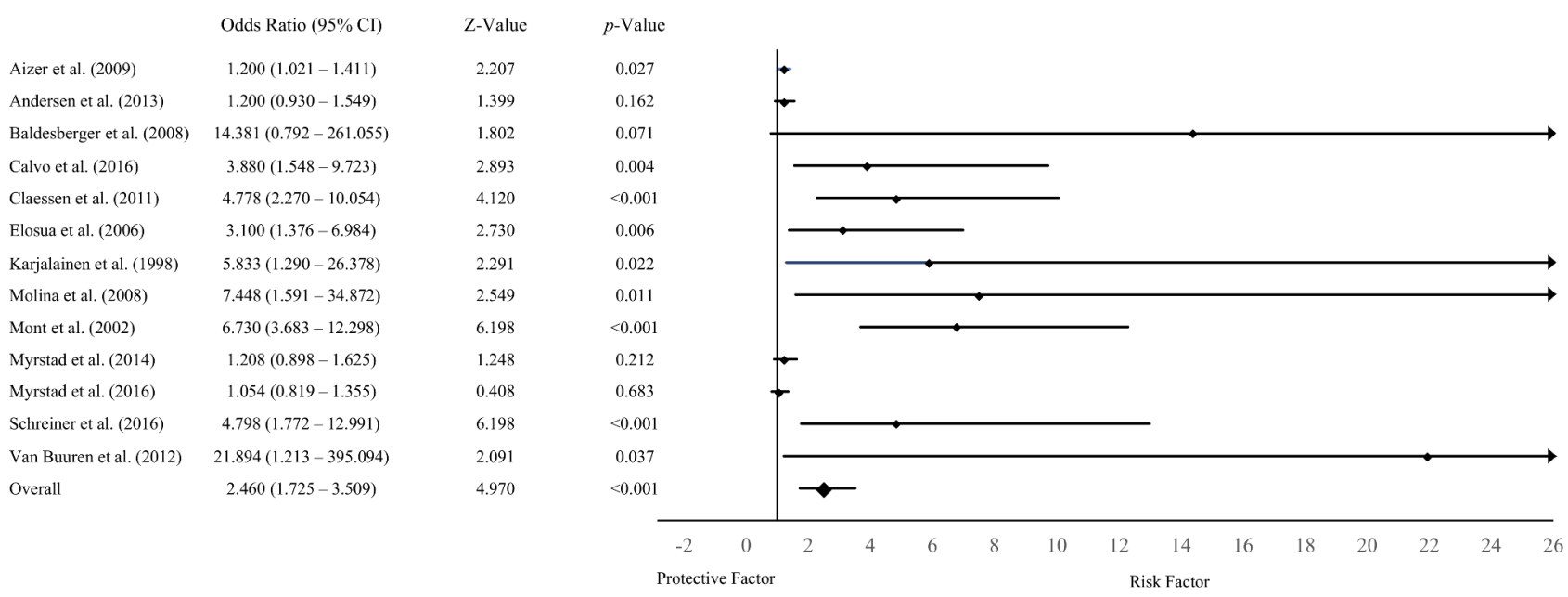

Figure 2 Random effects meta-analysis of AF risk in athlete's vs controls. AF, atrial fibrillation.

between study design and risk of AF, with athletes in case-control studies more likely to develop AF $(B=-1.3670, \mathrm{p}<0.001)$.

\section{DISCUSSION}

This meta-analysis aimed to quantify the risk of $\mathrm{AF}$ in athletes in an array of sporting activities compared with non-athlete controls. The results of the analysis indicate that the relative risk of $\mathrm{AF}$ is higher in athletes than in those not exercising or performing regular, non-competitive physical activity. Despite the increased risk observed among different study sizes, sport types, ages and exercise modalities, there remains a lack of high-quality studies with consistent methodologies to quantify the maximum safest regular 'dose' of exercise before AF risk becomes significant.

First, our OR, considering all primary studies, of 2.46 is much closer to the 'true' value as we included studies examining athletes, physically active individuals and sedentary populations, unlike other analyses. Additionally, the inclusion of studies involving athlete cohorts with common health conditions offers a more 'real world' estimation. Crucially, our research identifies a significant relationship between mode of exercise and risk of developing $\mathrm{AF}$, with mixed sport conferring a greater risk than endurance sport. Of the studies included in the analysis, three involved Nordic skiing, with these studies representing 36\% of the total weighting in the overall effect size. With this, it cannot be excluded that the remaining endurance sports included were comparatively underestimated in the analysis with Nordic skiing over-represented due to a greater quantity of published studies. Indeed, the observation that endurance sports correlate with $\mathrm{AF}$ is generally accepted. ${ }^{21}$ Mechanistically, Wilhelm ${ }^{13}$ indicates biatrial remodelling, dilation and fibrosis as crucial substrates to developing this pathology. However, Brugger et al ${ }^{22}$ suggested

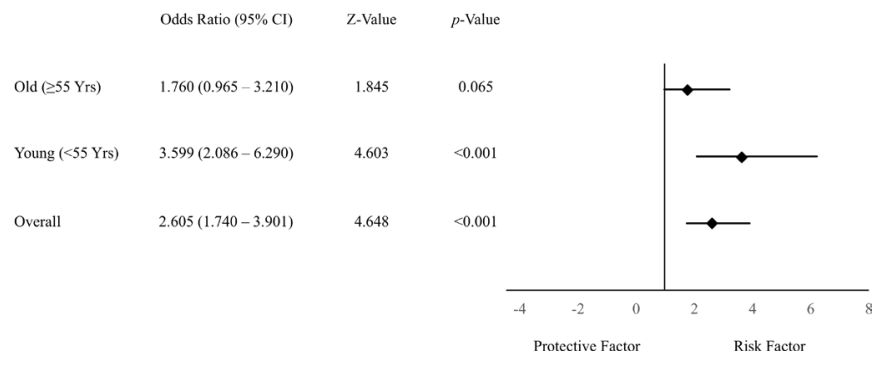

Figure 3 Subgroup analysis of age and the risk of atrial fibrillation. that mechanical and electrical remodelling of the atria seen with chronic endurance sport is functional and does not predispose individuals to a higher risk of AF. Despite this, current evidence does indicate a potential increase in $\mathrm{AF}$ risk with endurance exercise specifically. ${ }^{23}$ Separately, the association of 'mixed sports' with an increase in AF risk is difficult to interpret due to the broad range of sports analysed, complicating the process of elucidating true effects from specific training modalities. However, training volume may be an important risk factor for the development of AF and merits future research.

Our finding of a greater relative incidence of $\mathrm{AF}$ in younger athletes (OR: 3.60) echoes the results from Ayinde $e a^{15}$ who performed a similar meta-analysis. Additionally, Furlanello et $\mathrm{al}^{23}$ examined the relationship between $\mathrm{AF}$ risk and sport participation in elite and Olympic athletes, finding a significant prevalence among young (average age 24 years old), male athletes. However, the OR in older athletes was still 1.76 and much higher compared with non-athletes and thus must not be ignored. Investigating the mechanistic underpinning of this age-dependant risk, Hoogsteen et $a l^{24}$ discuss AF being triggered by adrenergic surges occurring during exercise in younger athletes ${ }^{25}$ whereas older athletes typically experience vagally induced $\mathrm{AF}$ after exercise, normally from secondary autonomic dysfunction. ${ }^{26}$ An interesting observation is that $\mathrm{AF}$ risk in the general population doubles at each decade after 50 years of age,

\section{Atrial Fibrillation in Athletes}

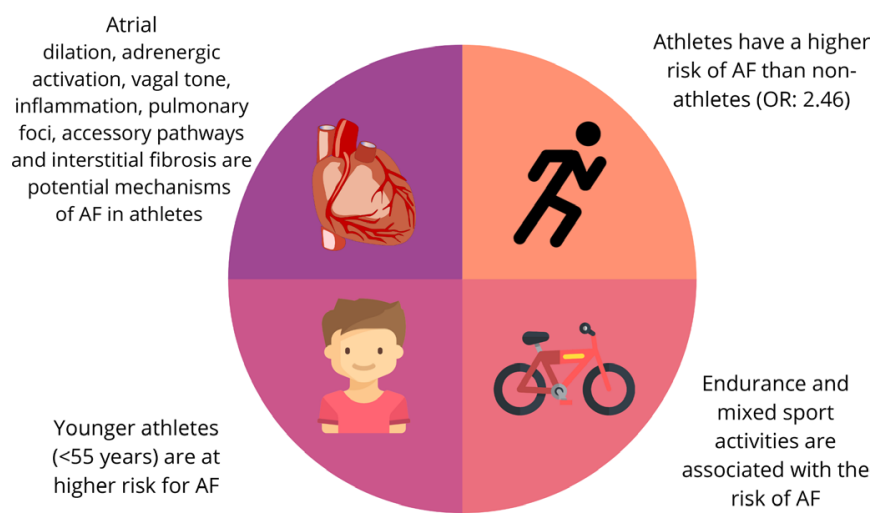

Figure 4 Central illustration of the main study outcomes. AF, atrial fibrillation. 
with a prevalence of up to $9 \%$ in $80-89$ year olds. ${ }^{8}$ With this, the lower association of AF in older athletes compared with younger athletes has been previously linked to the higher rates of $\mathrm{AF}$ in older individuals, independent from physical activity levels. ${ }^{27}$

Engagement with physical activity reduces CVD comorbidities that have been shown to increase the risk of AF through cumulative effects. ${ }^{28}$ In line with our inclusion criteria (studies where athletes train at least 3 hours a week), it is clear that once over national physical activity guidelines, AF prevalence begins to rise. ${ }^{29}$ However, it was not possible in these analyses to discern a specific duration and frequency threshold or type of exercise at which AF risk increased. Therefore, the current physical activity guidelines, whereby the benefits from the exercise outweigh the risks of any predisposition to AF, should be recommended. ${ }^{30}$

We also aimed to ascertain AF risk in all athletes, including samples representative of those with CVD risk factors, which are independently associated with AF risk among the general population. ${ }^{31}$ As such, we performed subanalyses on these CVD risk factors to understand their role in athlete and non-athlete $\mathrm{AF}$ prevalence. There was a greater prevalence of CVD in the non-athlete population, likely due to the cardiovascular protective role of physical activity; however, there was no statistically significant difference in the risk of AF in athletes and non-athletes with CVD risk factors. When CVD was excluded from analyses, the risk of $\mathrm{AF}$ in athletes was significantly greater compared with non-athletes (OR: 3.66).

\section{Study limitations}

One primary limitation of the present study is the possibility of residual confounding. The results of the included studies may be confounded in practice with studies applying different sets of adjusted variables. As detailed in Greenland and O'Rourke, ${ }^{32}$ the present analysis would have benefited from adjustment using external estimates in those studies which do not apply sufficient adjustment. Another issue with our study methodology arises around heterogeneity. Heterogeneity may develop from differences in study methodology, namely as our analyses sought to

\section{What is already known}

- Physical activity/exericse improves cardiovascular health and is associated with reduced morbidity and mortality.

- Prior research suggests there is a threshold beyond which exposure to increasing levels of exercise is associated with an increased risk of cardiovascular disease, including arrhythmias (U-shaped relationship).

- Current evidence does not allow for the identification of sport mode, which may predispose athletes to the greatest risk of atrial fibrillation.

\section{What are the new findings}

- Athletes have a significantly greater likelihood of developing atrial fibrillation compared with non-athlete controls.

- Our novel comparative analysis determined that mixed sport exercise modes convey the greatest risk of atrial fibrillation.

- Younger aged athletes have a greater relative risk of atrial fibrillation compared with older athletes; however, exercise dose parameters, including training and competition history, as well as potential gender differences for the risk of atrial fibrillation requires future research. include studies of both case-control and cohort design, which account for differences in between-trial heterogeneity. ${ }^{33}$ Our analysis was positive for publication bias when assessed using a qualitative risk of bias tool. ${ }^{34}$ Indeed, it is possible that studies with a null hypotheses of a significant relationship between AF and athletes were not published, alongside bias in study methodologies. However, it is prudent to express diligence in assigning heterogeneity only to methodology, as differences in diagnostic criteria may change between studies, masking the 'real' incidence of $\mathrm{AF}^{35}$ Importance is placed also in observing funnel plot asymmetry for qualitative demonstration of small studies exhibiting large effects, not just for bias. ${ }^{36}$ Indeed, $84 \%$ is well above an expected $\mathrm{I}^{2}$ value for a meta-analysis, probably owing to the differences in methodological parameters in the included studies. ${ }^{20}$ However, we wanted to include all primary research undertaken with athletes to determine a more clinically relevant aspect of risk estimation for practitioners and coaching staff. This analysis may also be limited by the possibility of sparse data bias in the pooled OR estimates, as indicated by the unconventional ORs and wide confidence limits produced by some studies. This is problematic as such bias can inflate the estimate of effect measures, which should be taken into account when interpreting the findings of the present analysis. ${ }^{37}$

This meta-analysis contained studies using both male and female athletes and male athletes alone. Due to the limited data in female athletes, it is difficult to discern the relative risk of $\mathrm{AF}$ by gender. In general, risk of AF in female athletes appears to be lower than in male athletes. ${ }^{29} 38$ It has been suggested that these differences in AF may be, in part, due to the proclivity for females to experience less atrial remodelling and electrocardiographic changes, alongside sex hormone differences which predispose females to higher vagal tone at rest and during exercise. ${ }^{29}$ However, future research is required, especially given the increased female participation in elite sports in recent years. Finally, due to the lack of available data, we were unable to provide information regarding training volume and the risk of $\mathrm{AF}$.

\section{CONCLUSION}

The risk of AF is greater in athletes compared with non-athletes. Mixed sports appear to confer a greater risk of AF. The risk of AF is relatively higher in younger athletes compared with older athletes, which is an association that should be investigated further (figure 4). Large prospective studies are needed to address current gaps in the literature including female athletes and the effect of years and volume of training.

Twitter William Newman @willwillnewman and Jamie O'Driscoll @JODriscoll9

Contributors WN and JO'D contributed to the conception and design of the study. WN, GP-W, JW, JE, SH, KK, MP, RS and JO'D contributed to the development of the search strategy. WN, JE, SH and JO'D conducted the systematic review. WN, GP-W, JE and JO'D completed the acquisition of data. WN, JE, SH and JO'D performed the data analysis. All authors assisted with the interpretation. WN, GP-W, JE and JO'D were the principal writers of the manuscript. All authors contributed to the drafting and revision of the final article. All authors approved the final submitted version of the manuscript.

Funding The authors have not declared a specific grant for this research from any funding agency in the public, commercial or not-for-profit sectors.

Competing interests None declared.

Patient consent for publication Not required.

Provenance and peer review Not commissioned; externally peer reviewed.

Supplemental material This content has been supplied by the author(s). It has not been vetted by BMJ Publishing Group Limited (BMJ) and may not have been peer-reviewed. Any opinions or recommendations discussed are solely those of the author(s) and are not endorsed by BMJ. BMJ disclaims all liability and responsibility arising from any reliance placed on the content. Where the content 
includes any translated material, BMJ does not warrant the accuracy and reliability of the translations (including but not limited to local regulations, clinical guidelines, terminology, drug names and drug dosages), and is not responsible for any error and/or omissions arising from translation and adaptation or otherwise.

\section{ORCID iD}

Jamie O'Driscoll http://orcid.org/0000-0002-5923-4798

\section{REFERENCES}

1 Kyu HH, Bachman VF, Alexander LT, et al. Physical activity and risk of breast cancer, colon cancer, diabetes, ischemic heart disease, and ischemic stroke events: systematic review and dose-response meta-analysis for the global burden of disease study 2013 BMJ 2016;354:i3857.

2 Myers J, McAuley P, Lavie CJ, et al. Physical activity and cardiorespiratory fitness as major markers of cardiovascular risk: their independent and interwoven importance to health status. Prog Cardiovasc Dis 2015;57:306-14.

3 Biddle S. Physical activity and mental health: evidence is growing. World Psychiatry 2016:15:176-7.

4 Arem H, Moore SC, Patel A, et al. Leisure time physical activity and mortality: a detailed pooled analysis of the dose-response relationship. JAMA Intern Med 2015;175:959-67.

5 Pathak RK, Elliott A, Middeldorp ME, et al. Impact of cardiorespiratory fitness on arrhythmia recurrence in obese individuals with atrial fibrillation: the CARDIO-FIT study. J Am Coll Cardiol 2015;66:985-96.

6 Mozaffarian D, Furberg CD, Psaty BM, et al. Physical activity and incidence of atrial fibrillation in older adults: the cardiovascular health study. Circulation 2008;118:800-7.

7 Adderley NJ, Ryan R, Nirantharakumar K, et al. Prevalence and treatment of atrial fibrillation in UK general practice from 2000 to 2016. Heart 2019;105:27-33.

8 Jones $C$, Pollit V, Fitzmaurice $D$, et al. The management of atrial fibrillation: summary of updated NICE guidance. BMJ 2014;348:93655.

9 Molina L, Mont L, Marrugat J, et al. Long-term endurance sport practice increases the incidence of lone atrial fibrillation in men: a follow-up study. Europace 2008:10:618-23.

10 La Gerche A, Schmied CM. Atrial fibrillation in athletes and the interplay between exercise and health. Eur Heart J 2013;34:3599-602.

11 Jin M-N, Yang P-S, Song C, et al. Physical activity and risk of atrial fibrillation: a nationwide cohort study in general population. Sci Rep 2019;9:13270.

12 Schoonderwoerd BA, Smit MD, Pen L, et al. New risk factors for atrial fibrillation: causes of 'not-so-Ione atrial fibrillation'. Europace 2008;10:668-73.

13 Wilhelm M. Atrial fibrillation in endurance athletes. Eur J Prev Cardio 2014:21:1040-8.

14 Abdulla J, Nielsen JR. Is the risk of atrial fibrillation higher in athletes than in the general population? A systematic review and meta-analysis. Europace 2009;11:1156-9.

15 Ayinde H, Schweizer ML, Crabb V, et al. Age modifies the risk of atrial fibrillation among athletes: a systematic literature review and meta-analysis. Int J Cardiol Heart Vasc 2018;18:25-9.

16 Moher D, Liberati A, Tetzlaff J, et al. Preferred reporting items for systematic reviews and meta-analyses: the PRISMA statement. Ann Intern Med 2009;151:264-9.
17 Pelliccia A, Sharma S, Gati S, et al. 2020 ESC guidelines on sports cardiology and exercise in patients with cardiovascular disease. Eur Heart J 2021:42:17-96.

18 Cameron AJ, Shaw JE, Zimmet PZ. The metabolic syndrome: prevalence in worldwide populations. Endocrinol Metab Clin North Am 2004;33:351-75.

19 Borenstein M, Hedges LV, Higgins JPT, et al. Introduction to meta-analysis. West Sussex, UK: Wiley, 2009

20 Huedo-Medina TB, Sánchez-Meca J, Marín-Martínez F, et al. Assessing heterogeneity in meta-analysis: Q statistic or 12 index? Psychol Methods 2006;11:193-206

21 Mont L, Sambola A, Brugada J, et al. Long-lasting sport practice and lone atrial fibrillation. Eur Heart J 2002;23:477-82.

22 Brugger $N$, Krause R, Carlen F, et al. Effect of lifetime endurance training on left atrial mechanical function and on the risk of atrial fibrillation. Int J Cardiol 2014;170:419-25.

23 Furlanello F, Bertoldi A, Dallago M, et al. Atrial fibrillation in elite athletes. J Cardiovasc Electrophysiol 1998;9:S63-8.

24 Hoogsteen J, Bennekers JH, van der Wall EE, et al. Recommendations and cardiological evaluation of athletes with arrhythmias: Part 1. Neth Heart J 2004:12:157-64.

25 Workman AJ. Cardiac adrenergic control and atrial fibrillation. Naunyn Schmiedebergs Arch Pharmacol 2010:381:235-49.

26 Coumel P. Paroxysmal atrial fibrillation: a disorder of autonomic tone? Eur Heart $J$ 1994;15(Suppl A):9-16.

27 Brunetti ND, Santoro F, Correale M, et al. Incidence of atrial fibrillation is associated with age and gender in subjects practicing physical exercise: a meta-analysis and meta-regression analysis. Int J Cardiol 2016;221:1056-60.

28 American College of Sports Medicine, Chodzko-Zajko WJ, Proctor DN, et al. American College of sports medicine position stand. exercise and physical activity for older adults. Med Sci Sports Exerc 2009;41:1510-30.

29 Myrstad M, Aarønæs M, Graff-Iversen S, et al. Does endurance exercise cause atrial fibrillation in women? Int J Cardiol 2015;184:431-2.

30 Elliott AD, Linz D, Mishima R, et al. Association between physical activity and risk of incident arrhythmias in 402406 individuals: evidence from the UK Biobank cohort. Eur Heart J 2020;41:1479-86.

31 Benjamin EJ, Levy D, Vaziri SM, et al. Independent risk factors for atrial fibrillation in a population-based cohort. The Framingham heart study. JAMA 1994;271:840-4.

32 Greenland S, O'Rourke K. Meta-analysis. In: Rothman KJ, Greenland S, Lash T, eds. Modern epidemiology. 3rd edn. Philadelphia, PA: Lippincott Williams \& Wilkins, 2008.

33 Thompson SG, Sharp SJ. Explaining heterogeneity in meta-analysis: a comparison of methods. Stat Med 1999;18:2693-708.

34 Egger M, Davey Smith G, Schneider M, et al. Bias in meta-analysis detected by a simple, graphical test. BMJ 1997;315:629-34.

35 Higgins JPT, Thompson SG. Quantifying heterogeneity in a meta-analysis. Stat Med 2002;21:1539-58.

36 Sterne JA, Egger M, Smith GD. Systematic reviews in health care: investigating and dealing with publication and other biases in meta-analysis. BMJ 2001;323:101-5.

37 Greenland S, Mansournia MA, Altman DG. Sparse data bias: a problem hiding in plain sight. BMJ;2016:i1981.

38 Svedberg N, Sundström J, James S, et al. Long-Term incidence of atrial fibrillation and stroke among Cross-Country skiers. Circulation 2019;140:910-20. 